\title{
The advancement in magnetic nanoparticles for the diagnosis of lung cancer and its monitoring
}

\author{
Ayushi Rastogi ${ }^{1 *}$, Fanindra Pandey $^{2 *}$, Pankaj Tripathi ${ }^{1 *}$, Rajiv Manohar ${ }^{{ }^{*}}$, Shri Singh $^{2^{*}}$ \\ ${ }^{1}$ Department of Physics, University of Lucknow, UP- 226007, India \\ rastogiayu19@gmail.com; pankajtripathi19@gmail.com; rajiv.manohar@gmail.com \\ ${ }^{2}$ Department of Physics, Banaras Hindu University, Varanasi, UP - 221005, India \\ Fanindrapatipandey143@gmail.com; srisingh@bhu.ac.in \\ Correspondence: Ayushi, Fanindra, Pankaj, Rajiv, Shri
}

\begin{abstract}
There are numerous challenges involved in the diagnosis and treatment of lung cancer. Globally, majority of people suffers from cancerous disease involving throat cancer, lung cancer, stomach cancer, cancerous brain tumor. Among these the most common ones is the lung cancer or lung carcinoma. The leading cause of the lung cancer is the smoking. Around $80 \%$ to $90 \%$ of deaths are caused due to Non-small cell lung cancer (NSCLC). The inadequate diagnostic techniques and low chances for the survival of lung cancer patients results from the lack of an early prognosis and incompetency in traditional therapies. However, such challenges involved in the prognosis and treatment of lung cancer are on decline with the progression in magnetic nanoparticle (MNP) technology. Many break-through discoveries and inventions have been made in the field of cancer therapy by using magnetic nanoparticles. The implication of nanotechnology has led to the recent advancement in nanomedicine field. This has encouraged the improvement in different therapeutic and diagnosis strategies employing nanotechnology. The generation of immense technological benefits for nanoparticles systems has been accredited to its remarkable nanoscale physico-chemical properties. This in turns provides the early detection of lung cancer and active drugs delivery for an improved theranostics strategy. The present review provides a general idea of the current progression in the therapeutic and prognosis purpose of magnetic nanoparticles. Further, we disclose the development in the lung cancer theranostics by functionalization of magnetic nanoparticles. The established importance of magnetic nanoparticles in the theranostics centers for lung cancer has been revealed in this paper. The challenges existing in the theranostics of lung cancer are addressed through the functioning of magnetic nanoparticles in the process.

Keywords: Lung Cancer; Magnetic nanoparticles; Detection and monitoring; Theranostics
\end{abstract}

\section{Introduction}

The multiplication of cells which consumes oxygen and other essential nutrients from the living cells defines as the cancer. The development of secondary tumor enlargement arises from the cancerous cells which are dodged from these living cells. The cancer cells spreads to other tissues or body parts through the bloodstream or lymph nodes. The removal of this tumour from the body can be possible in its early stage of growth [1]. An increased risk of metastasis commonly causes the death of patients. Metastasis of various cancers like lungs, bone and liver can be cured in some situations. However, the treatment does not cure the cancer as it can only curb its growth and thus reduces its symptoms. It is possible for the cancer patients to live life for several months or years even after the progression of metastatic disease. The tumors can be malignant or non malignant. The non malignant tumors can be cured simply by removing it as in 
non malignancy the cells are non cancerous which does not gets multiply on surgery. In contrast to this, the malignant tumors cannot be cured completely due to malignancy. The malignancy involves the hyper replication of cancerous cells with its rapid development in the lymph nodes or bloodstream. The metastasis cancer can be cured to some extent. There are nearly 200 kinds of cancers that are prevailing with different symptoms. The root cause of the cancerous diseases is linked with diverse living factors like age, daily consumption, genetic and lifestyle causes. In 2011, UK Cancer Research group reported that among the existing cancers, lung cancer is the most broadly spread one in a global platform $[2,3]$.

\subsection{Past to Present Scenario}

The Lung disease is mainly classified as: small cell lung cancer (SCLC) and non-small cell lung cancer (NSCLC). The contribution of SCLC is $13 \%$ out of the total lung cancer cases and NSCLC has $85 \%$ contribution out of total reported cases. Collins et al., reported SCLC as vastly metastatic which is confined to preliminary and secondarily bronchi. NSCLC being simple to identify and frequently arises in the lung peripheral tissues [4]. According to Howlader et al., NSCLC has been additionally classified depending on histopathology and categorized as squamous cell carcinoma, adenocarcinoma and large cell carcinoma [5]. The increase in the number of mortality rate examined amid lung carcinogenic patients has been ascribed to the delay in cancer prognosis. Bjerager et al., and Mackillop et al., reported the misdiagnosis or unidentification of the lung cancer arises from the merging of the disease symptoms with other respiratory problems such as Emphysema and chronic bronchitis (COPD), chronic obstructive pulmonary disease $[6,7]$. Aberle et al., suggested that low- dose computed tomography LDCT screening test for evaluating the total mortality rate has not been statistically considerable [8]. Wildstein et al., and Goulart et al., describes the limited reliability of LDCT as the screening test for lung cancer because it involves excessive expenditure [9, 10]. The exposure of harmful radiations during LDCT test involves high risk to our body that has been elaborated by Brenner, McCunney and Li [11, 12]. As pointed out by Anari et al., [13] the decline in the mortality rate of cancer has not been feasible even slightly despite of the considerable efforts made in the last 50 years. The report of American Cancer Society on new evolving cases of lung cancer anticipated over 221,200 patients and 158,040 death in the year 2015 in the United States $[14,15]$. Approximately $85 \%$ of them had NSCLC lung cancer while other $15 \%$ had SCLC cancer. The dependence of the survival rate in lung cancer patients is preliminary based on early prognosis. The most general therapy which is being preferred for the treatment of lung cancer is the removal of the tumor tissue by expensive surgeries [16, 17]. The issue related to the treatment of cancer has not yet been resolved completely and even the clinically used theranostics strategies and modalities are allied with a meager outcome. The drug therapies used to treat cancerous cells normally become impervious. This causes barely $<20 \%$ of five-year total survival rate. Amid all the currently accessible cancer theranostics strategies known till now, chemotherapy is mainly the most extensively used ones for the lung cancer. The retardation in the clinical success of chemotherapy sections has been mainly related to the inadequate drug delivery in the tumor tissue. By far, the foremost reason of increasing cancinoma fatality rate worldwide is the bronchogenic/lung carcinoma predominantly, NSCLC. It is the secondary widespread cancinoma disease in both men and women. As elaborated in the report of Munaweera et al. and Tabatabaei Mirakabad et al. [18, 19], In the United States, the Lung cancer is the imperative cause of cancinoma associated deaths. Later in 2017, Wong et al., [20] found that the disorder predominance and mortality rates are higher in the districts which are 
developing or under-developed zones of the world. It is absolutely interrelated with the socioeconomic concern of the affected inhabitants. In the Southeast Asia, the bronchogenic carcinoma is contemplated to be the severe public health apprehension and is a primary ground of mortality. By the year 2018 Bray et al., [14] noticed that the syndrome has a global contribution of about $11.6 \%$ of all the recent prognoses carcinoma cases and $18.4 \%$ of overall mortality rate. The exaggerated response of our immunity system to drugs delivered to treat lung cancer is one of the leading causes of carcinoma associated deaths worldwide. Lung cancer is the most widespread in men accounted for projected 154,050 deaths in 2018 globally [14, 15]. As per the GLOBOCAN, a project of the International Agency for Research on Cancer (IARC), accounted about 18.1 million new carcinoma patients that would have been diagnosed in 2018 [14]. The origin of this outrageous disease comprises of vastly $85 \%$ to tobacco smoking for long term [21]. Of all the lung cancers existing so far NSCLC type accounts to about 85\%. Globally, a prognostic of 29.5 million mortality rate has been predicted by 2040 as per the report of world health organization (WHO). The WHO statistics data for the year 2019 reveals cancer being the secondary principal basis of globally recorded deaths [22]. Therefore, there is requirement of recognizing specific biomarkers to infectious diseases which are competent enough to detect the occurrence of malignant cells at premature stage. This might provide a support to ease mortality rates. In the latest case on Dec. $31^{\text {st }} 2019$, china informed WHO about the occurrence of several severe cases of pneumonia of unknown cause happening in Wuhan City in Hubei district. Later on $9^{\text {th }}$ Jan. 2020, WHO recorded the statement that the Chinese researchers have made a primary revelation of a novel virus named corona virus. Severe acute respiratory syndrome (SARS) corona virus disease 2019 (COVID- 19) - a pandemic has recorded mortality rate of globally. This virus is still spreading with increasing mortality rates day by day. Till now no complete cure has been discovered so far for this disease. Even today lot of efforts have been put forward by various world top scientists, experts, medical care research institutes and universities in order to invent the vaccine that could eradicate this virus completely so that people can live their life in a normal way. Thus, for various researchers in virology, medical, healthcare fields the future course of direction has now shifted to the main motive of fighting against the pandemic SARS COVID19. In these critical times, retrieving the authentic and reliable information is of paramount significance. For the benefit of researchers, scientists and academicians we are now composing the most pertinent parts of our coverage in the form of an article that we hope will be a handy guide to good health practices as well as in fighting the cancer and generating a guide to fight against novel COVID- 19.

\subsection{Global Challenges involved in Lung Cancer}

On the line of diverse kind of cancers in the globe, the Lung carcinoma counts to second number in both genders (feminine and masculine). The treatment of lung carcinoma involves different operational and non operational therapeutic methods including photodynamic therapy, radiotherapy and chemotherapy. However, most of these practices are generally expensive, complicated and devoid of patient conformity. The inadequate drug delivery and inability to combat with tumor tissues in chemotherapy sections may lead to lack of sufficient drugs targeting to tumor sites. The recovery rate in the lung cancer patients are low and even the recovered patients remains at the exposure of recurrence. The detection of metastasis is complex by usual imageology and histopathology methods. Therefore, more insightful and precise techniques are required to diagnose lung cancer in the early stages and be acquainted with patients at utmost threat for relapse. To overcome the problems related to chemotherapy, the 
synthesization of different kinds of organic and inorganic nanoparticles is considered in combating the lung cancers. To meet these theranostics challenges, the high concentrations of anti-cancer drugs are continuously administered in systemic chemotherapy sections which cause adverse side effects in patient body. The chemotherapy based carcinoma treatments involves the penetration of various powerful cytotoxic drugs in tumor tissues however, its penetration may cause adverse side effects on other healthy non cancerous body tissues. This is the one of the severe side effect observed in chemotherapy cancer treatments $[23,24]$. Therefore, there is an urgent need for novel inventions and discoveries related to theranostics modalities for more efficient therapeutic for lung cancer. The optimistic result of lung cancer treatment is sturdily associated with the early diagnosis. Thus, the strong technologies and machineries requirement could offer an early diagnosis of cancer related diseases. The perception of early diagnosis is directly extensive to large community screening, and then, it is stoutly associated with the low cost and non invasiveness.

\subsection{Magnetic Nanoparticles in Lung Cancer}

The relevance of nanomaterials have laid down the foundation for emerging usefulness in the areas of biomedicine and imaging technologies. Nanomaterials are profoundly practical for its applications in tumor targeting and its visualization. This allows the early diagnosis of lung cancer. Another medicinal function of nanomaterials is delivering the drugs in the targeted regions. The improvement in the therapeutic efficiency is possible through these intelligent nanocarriers which tailor the transportation of anticancer drugs to the targeted regions where they are liberated without impairment of healthy cells. In the oncology domains magnetic nanoparticles (MNPs) have an important contribution in the cancer theranostics, screening, targeted drug delivery and healing procedures. The implication of MNPs is widely practical in tumor targeting process for the reason that, tumor visual technologies have unlocked the opportunities for early diagnosis of the cancerous disease. The superparamagnetic nature of iron oxide nanoparticles i.e., SPIONs have been typically utilized as distinguished agents in case of magneto-acoustic tomography (MAT), carcinoma screening for magnetic resonance imaging (MRI), near-infrared (NIR) imaging and computed tomography (CT). Moreover, another application of MNPs which cannot be ignore is, the targeted drug delivery where the external magnetic field (EMF) is applied to detect the precise location of the targeted tumor tissues by the use of MNPs as drug carriers for in vivo method. The functionalization of MNPs with antibodies is extremely important for detecting the particular targeted cells collectively with chemotherapeutic drugs. The application of MNPs can be functional in carcinoma therapy by photodynamic therapy (PDT), magnetically induced hyperthermia (MHT) and photothermal therapy (PTT). These strategies are employed in the oncology field of medical sciences. However, the paramount theranostics consequence is generally certain by their combined effect. This has been attributed to the multiple functioning of MNPs due to its modular design. For example, magnetic resonance imaging in Figure 1 (A) has been relevant for the early prognosis of carcinoma disease. Thus, the combination of the chemotherapeutic treatment with magnetic resonance imaging or MRI could generate improved and faster outcomes [25]. The protocol involved in the synthesization process controls and modifies the ultimate characteristic features of MNPs. The most significant characteristics of MNPs for biomedical relevance includes enhanced magnetic moment, super paramagnetism, small particle diameter, large specific surface area and magnetocaloric effect that might possibly be fundamentally functionalized [25]. The core region of MNPs influences its magnetic properties. Therefore, the superparamagnetic property is normally practical for the MNPs with the size up to $100 \mathrm{~nm}$ and hence it is dependent 
on the nanoparticle dimensions. The clustering of MNPs is prevented by applying electromagnetic field (EMF). The EMF magnetizes the nanoparticle and this magnetization is lost as soon as field is removed thus precluding the MNPs aggregation [25]. Some MNPs exhibits magnetocaloric effect in which there is change in the temperature of MNPs due to the subsistence of EMF. Now, the combination of magnetocaloric effect with a large surface-tovolume ratio of MNPs permits the proficient heat exchange with the environment. This makes it possible to generate the newest carcinoma therapeutic approach, explicitly known as "hyperthermia". The exceptional properties of MNPs are entirely subjugated in tissue labeling and targeted drug delivery sites as depicted in Figure 1(B). In this figure, the drugs is delivered to the specific target cell by suitable positioning of the magnetic field exterior to the body parts and adopting a in vivo method of transportation [25]. The absorption of heat produced from the electromagnetic wave (EMW) in the alternate cycles by MNPs, makes this magnetic substance well considerable in the field of bioscience. The small size of MNPs plays the important role. As previously illustrated, an immense benefit of MNPs is their small dimension. The MNPs dimension is generally less or analogous to biological units varying from several nanometers for genes and proteins, to hundreds of nanometers for viruses and up to $100 \mathrm{~nm}$ for cells. The small size of MNPs is advantageous for their better diffusion and precise distribution capabilities in tissues or in the environs of the targeted sites. Additionally, the adjustment in a variety of processes involved in the development of magnetic features of MNPs as well as to envisage their in vivo behavior is required. This has been possible by changing parameters such as composition, size, surface functionalization or morphology. The characteristic properties of most frequently employed MNPs like iron oxide nanoparticles and particularly magnetite $\left(\mathrm{Fe}_{3} \mathrm{O}_{4}\right)$ and its oxidized form maghemite $\left(-\mathrm{Fe}_{2} \mathrm{O}_{3}\right)$ have fascinated consideration essentially attributed to their low toxicity, biocompatibility and cost, simplistic synthesization, as well as their explicit optical and magnetic properties. This can be subjugated in fabrication of different microsystems and medical devices.

Moreover, the relevance of magnetic nanoparticles in the area of carcinoma biomedicine are connected to their implication in magnetic resonance imaging as distinguished agents and in cancerous thermotherapy also known as hyperthermia [25], as heating intermediaries (Figure 1 (C)). Furthermore, unique functions and characteristics of MNPs as podium of antibodies immobilization or aptamers for development of biosensors have been projected (Figure 1 (D, E)). The immobilization of affinity ligands such as hEGF, aptamers, lectin, folic acid at the MNPs surface has been performed in order to direct them in the tumors. This facilitated the accumulation of MNPs in a specific cells or tissues location (Figure 1(F)) [25 - 28]. Henceforth, the combination of these stratagems with guided drug delivery and magnetic hyperthermia facilitated a cooperative or synergetic impact in the proficient cure of cancerous diseases. 


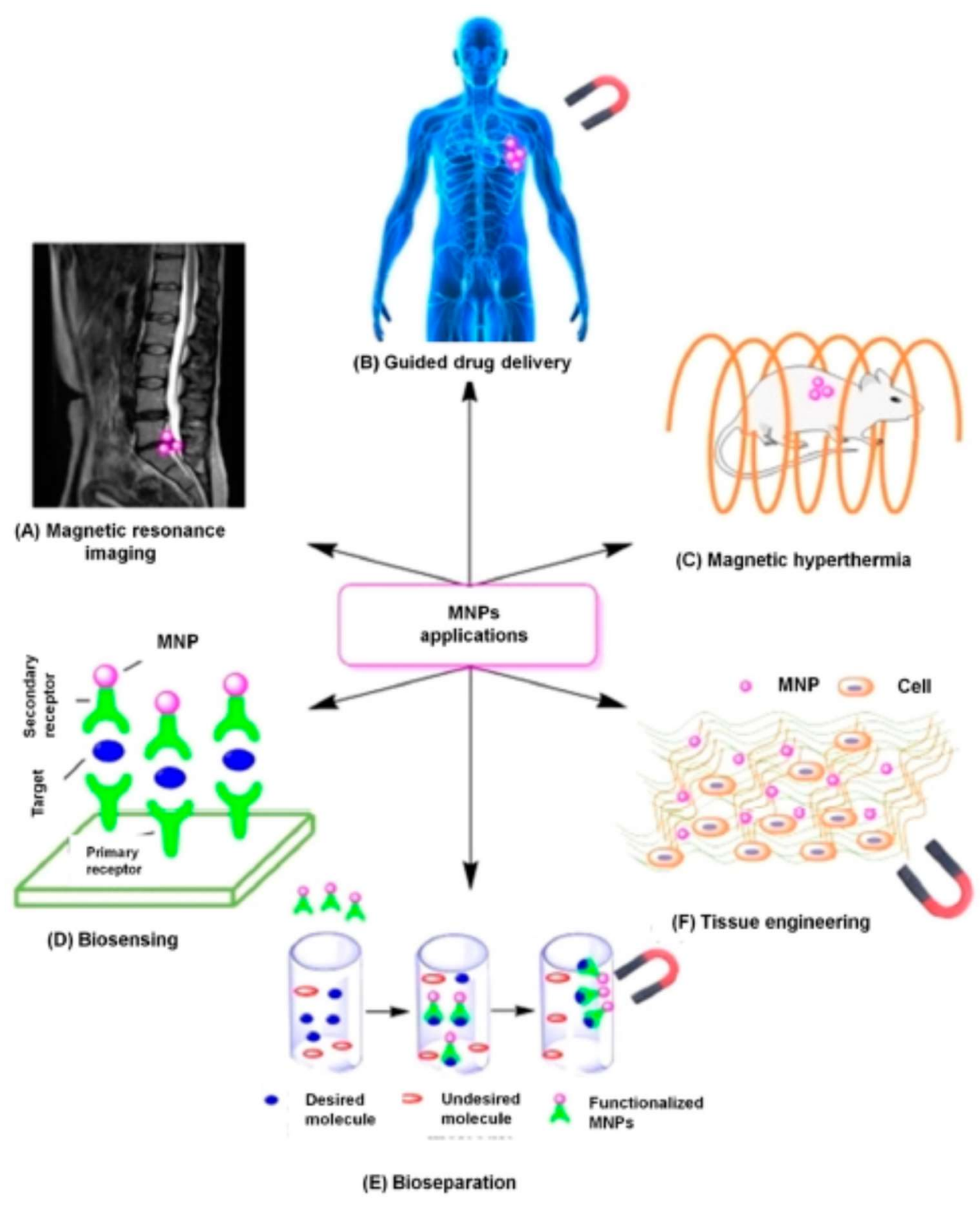

Figure 1 Diagrammatic illustration of potential relevance of magnetic nanoparticles in biomedicine: (A) MRI magnetic resonance imaging, (B) Targeted/guided drug delivery, (C) magnetic hyperthermia, (D) Biosensing, (E) Bioseparation, and (F) Tissue engineering. [26]

\subsection{Synthesization of Functionalized Magnetic Nanoparticles}

The treatment of cancerous cells lines with silicate nanoparticles (NPs) using vanadium, europium or yttrium core and anticancerous medicine doxorubicin released in $\mathrm{pH}$ - dependent way has been illustrated by Shanta Singh et al. [29]. The attainment of cytotoxic effect after the addition of iron oxide nanoparticles has been achievable by the application of an alternating magnetic field [29]. An advanced uptake and large toxicity in the medication of glioma cells has been managed by Fang et al., employing formulation of iron oxide nanoparticles, poly- $\beta$ amino-ester-copolymer and anticancer drug doxorubicin which was not possible with the pure iron oxide nanoparticles [30]. The functionalization of MNPs such as iron oxide by coating it with $\beta$-cyclodextrin and F127 polymer results in the modification of encapsulated drug release 
as reported by Yallapu et al. [31]. This produces an advanced curative efficiency against cancerous disease [31]. The implication of nickel Ni-based MNPs by Guo et al. furnishes daunorubicin with an advanced cytotoxicity that opposes in vitro leukemia cells [32]. A particular structure of targeted drug delivery involves magnetic drug targeting (MDT) in order to cure cancer. This therapy uses chemotherapeutic mediators reliably related with the superparamagnetic iron oxide NPs. The resultant ferrofluid solution is directly supplied to the tumor by injecting it into an artery. The application of external magnetic field concentrates the particles and the attached drugs in the tumor (Figure 2) [33]. The in vitro relevance of these magnetic iron oxide NPs have also been tested. No toxicity was found in therapeutically significant concentrations [34]. The development of sturdy magnetic field of gradient up to 72 $\mathrm{T} / \mathrm{m}$ at the pole tip by using an electromagnet and a high magnetic flux density was specially employed for this principle [35]. In an ex vivo arterial model, the investigation of the flow and circulation of NPs were elaborated in detail under various predicted conditions [36, 37].

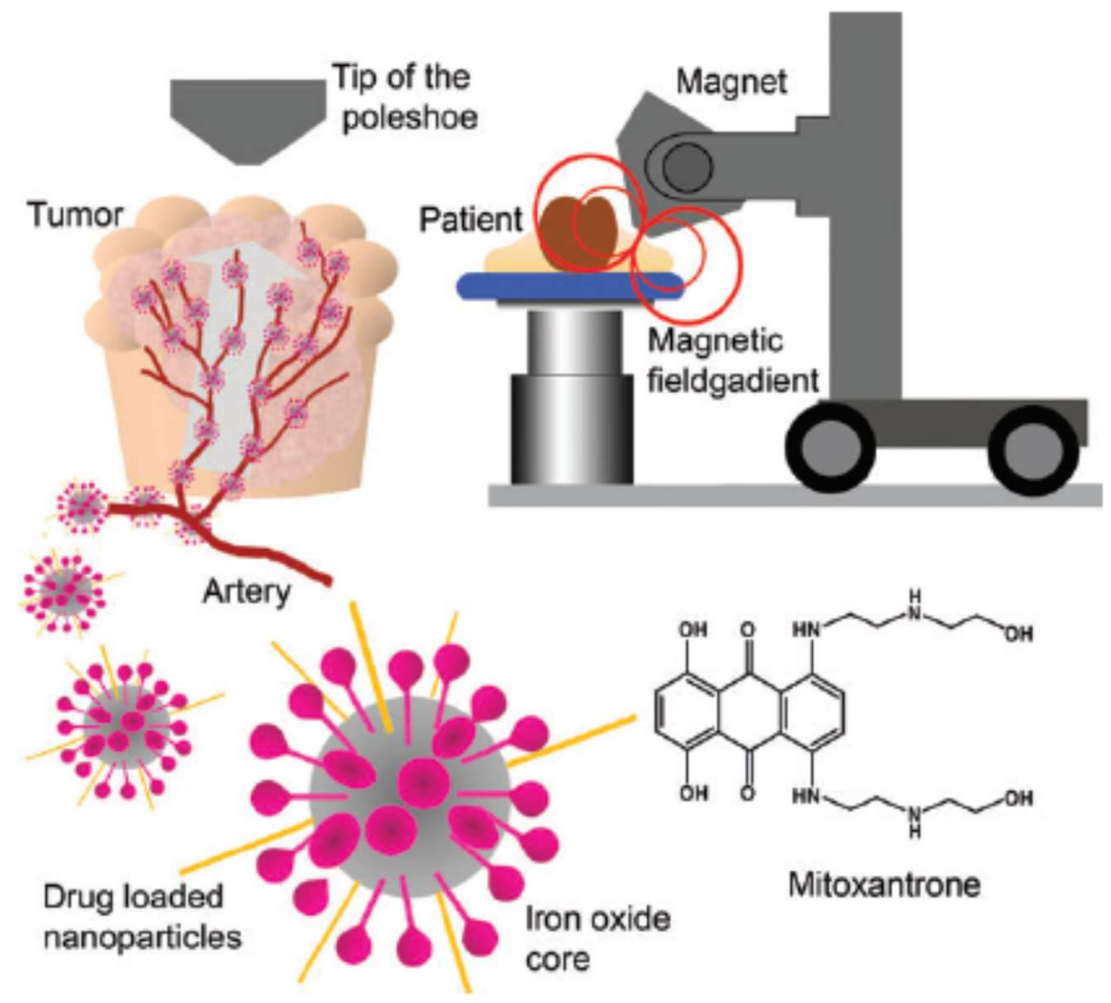

Figure 2 Magnetic drug targeting (MDT) principle. The functionalization of MNPs iron oxide with a chemotherapeutic mediator and its injection directly applied into the tumor arterial supply. The appliance of sturdy external magnetic field generates their improvement in the tumor [33].

In the reported work of Wang et al. [38], there is effective coupling of synthesized MNPs with pan cytokeratin antibody abbreviated as pan-ck $\mathrm{Ab}$, together with other two types of synthesized quantum dots were in conjugation with Lunx and SP- A antibodies. The identification of the circulating tumor cells of NSCLC patients assembled by MNPs coupled with pan cytokeratin (MNP-pan-ck) was done by the simultaneous use of Quantum dots with doublelabeled antibodies. Henceforth, a novel and innovative methodology for testing micrometastases of lung carcinoma in peripheral blood by MNPs and quantum dots was productively engineered. 
The synthesization process of MNPs involves various significant processes as proposed by different researchers. A lot of these processes presented extraordinary properties like monodispersity, shape control, stabilization and fabrication on a vast scale like co-precipitation or hydrothermal synthesis and sol-gel process. Novel and modern techniques were dealt with: thermal decomposition, microemulsion and sonolysis biosynthesis [25, 39]. The choice of the best synthesization route is dependent mostly on the type of MNPs an individual desires to get. Following is the classification of MNPs depending on their composition:

1. Iron oxide nanoparticles or oxides (ferrites),

2. Hematite $\left(-\mathrm{Fe}_{2} \mathrm{O}_{3}\right)$,

3. Maghemite $\left(-\mathrm{Fe}_{2} \mathrm{O}_{3}\right)$ and

4. Magnetite $\left(\mathrm{Fe}_{3} \mathrm{O}_{4}\right)$.

The easy surface functionalization of NPs with different compounds has been involved in the biomedical applications which are responsible for its enhanced stability in suspension form (example: silica, surfactants) [40]. Metallic NPs with a metallic core are further appropriate for biomedical relevance. This has been associated with their large magnetic moment in comparison to their oxides. The stated limitations are pyrophoric property and existence of high reactivity to chemical changes and other oxidizing agents. The surface modification of MNPs has also been possible by using ferrites as shell layer. The cores of chemically inert MNPs have been enveloped by a silica shell through covalent bonding for further functionalization process. In case of metallic NPs, the core is enveloped by shell layer made up of well developed metals, polymers or customized surfactants [40].

Table 1 It elaborates numerous methodologies for the synthesization of MNPs [25, 41].

\begin{tabular}{|c|c|c|}
\hline Method & Details & Reference \\
\hline Co-precipitation & $\begin{array}{l}\text { The most simplistic and well proficient } \\
\text { method for synthesization of MNPs. It involves } \\
\text { - iron oxides NPs extracted from aqueous } \\
\text { solution of } \mathrm{Fe}^{2}+/ \mathrm{Fe}^{3}+\text { salts; } \\
\text { - Numerous parameters are required to be } \\
\text { well-known like } \mathrm{Fe}^{2}+/ \mathrm{Fe}^{3}+\text { ratio, pH level, solvent } \\
\text { nature, surrounding temperature, etc; }\end{array}$ & {$[40]$} \\
\hline $\begin{array}{l}\text { Thermal and } \\
\text { Hydrothermal Decomposition }\end{array}$ & $\begin{array}{l}\text {-synthesis of MNPs in aqueous solution } \\
\text { under high temperature and pressure conditions; } \\
\text {-improvement in the rate of nucleation } \\
\text { and speed up the development and growth of new } \\
\text { generated particles; } \\
\text { - the most general used reactions involves } \\
\text { hydrolysis and oxidation reaction } \\
\text { - Alternative means is the neutralization } \\
\text { reaction of hybrid metallic hydroxides; } \\
\text { - advantageous in production of particles } \\
\text { of small dimensions; }\end{array}$ & {$[42]$} \\
\hline Sol-Gel Processes & $\begin{array}{l}- \text { Generation of sol of NPs by } \\
\text { hydroxylation and condensation reactions. } \\
\text { - Generation of } 3 \text {-D network metallic } \\
\text { oxide gel by condensation reaction } \\
\text { - Temperature assisted process results in }\end{array}$ & [39] \\
\hline
\end{tabular}




\begin{tabular}{|c|c|c|}
\hline & $\begin{array}{l}\text { formation of gel in crystalline form. } \\
\qquad \text {-Numerous parameters require to be well } \\
\text { known such as salts concentrations, pH level, } \\
\text { temperature, solvent nature, precursors ratio, etc.; } \\
\text { - Addition of surfactants influences the } \\
\text { synthesization of three dimensional gel network; } \\
\quad \text { - main limitation in this method is the } \\
\text { occurrence of gel coagulation. }\end{array}$ & \\
\hline $\begin{array}{l}\text { Microemulsion } \\
\text { and } \\
\text { Inverse Micelles }\end{array}$ & $\begin{array}{l}\text {-This method is used for the synthesis of } \\
\text { specific type of } \mathrm{MNPs} \text { i.e., } \mathrm{MFe}_{2} \mathrm{O}_{4} \text {, where } \mathrm{M} \\
\text { stands for } \mathrm{Cu}, \mathrm{Mn}, \mathrm{Ni}, \mathrm{Co}, \mathrm{Cd}, \mathrm{Zn}, \mathrm{Mg} \text {, etc., major } \\
\text { magnetic elements for relevance in electronic } \\
\text { applications; } \\
\text { - the facile tailoring of the size and shape } \\
\text { of } \mathrm{MFe}_{2} \mathrm{O}_{4} \text { depends on different functional } \\
\text { parameters; } \\
\quad \text {-main limitations: inconsiderate } \\
\text { experimental circumstances like large amount of } \\
\text { solvent consumption, narrow working window, } \\
\text { low NPs yield; }\end{array}$ & [43] \\
\hline Biosynthesis & $\begin{array}{l}\text { - Generation of biocompatible MNPs by } \\
\text { environmental affable method; } \\
\text { - biosynthesis of MNPs by using reducing } \\
\text { agents like bacteria, microbial enzymes, plant } \\
\text { phytochemicals and magnetotactic bacteria; } \\
\quad \text { - main Limitations: the clear elucidation } \\
\text { of biological mechanism for the synthesization of } \\
\text { MNPs has not yet been done by this method; } \\
\text { modulation of required parameters cannot be } \\
\text { performed for shape- and size controlled synthesis } \\
\text { of NPs; }\end{array}$ & [44] \\
\hline Sonolysis & $\begin{array}{l}\text {-Method based on high intensity } \\
\text { ultrasound; } \\
\text { - the commutative expansion and } \\
\text { compression in acoustic waves give rise to } \\
\text { oscillating cavities of varying size; } \\
\text {-the accumulation of an ultrasonic energy } \\
\text { by the oscillating fissure of a definite size; } \\
\text { - Benefits: mild experimental } \\
\text { circumstances such as pressure, reaction time } \\
\text { temperature; }\end{array}$ & {$[45]$} \\
\hline Spray/laser Pyrolysis & $\begin{array}{l}\text {-Occurrence of particles nucleation by } \\
\text { condensation after sprinkling an iron Fe salt } \\
\text { suspension into a hot air or a laser beam; } \\
\text { - this method follows temperature } \\
\text { controlled decomposition of the generated } \\
\text { particles; } \\
\text { - Benefits: production of efficiently small } \\
\text { particles with size approx. 5-60 nm range; } \\
\text {-main Limitations: More complex, }\end{array}$ & {$[46]$} \\
\hline
\end{tabular}


sophisticated and costly instruments, interference of oxygen and other gases;

There are several reported works on the functionalization of MNPs surface by using different chemical agents [47- 50]. This has been schematically represented in Figure 3 (a). Moreover, a summarized illustration on the kinds, functionalities and shapes for MNPs so that they are exploited as drug delivery carriers in cancerous therapeutic along with the demonstration of biophysicochemical properties has been provided in Figure 3 (b).

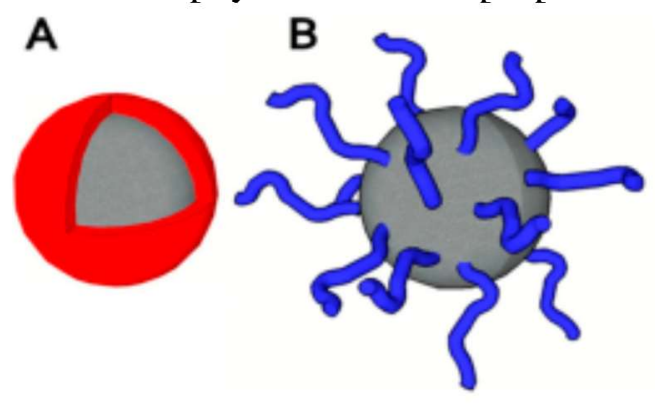

C
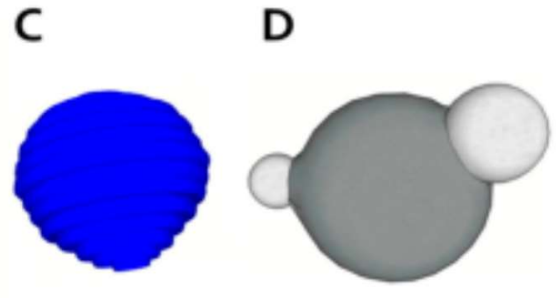

E

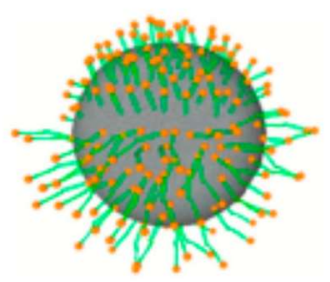

(a)

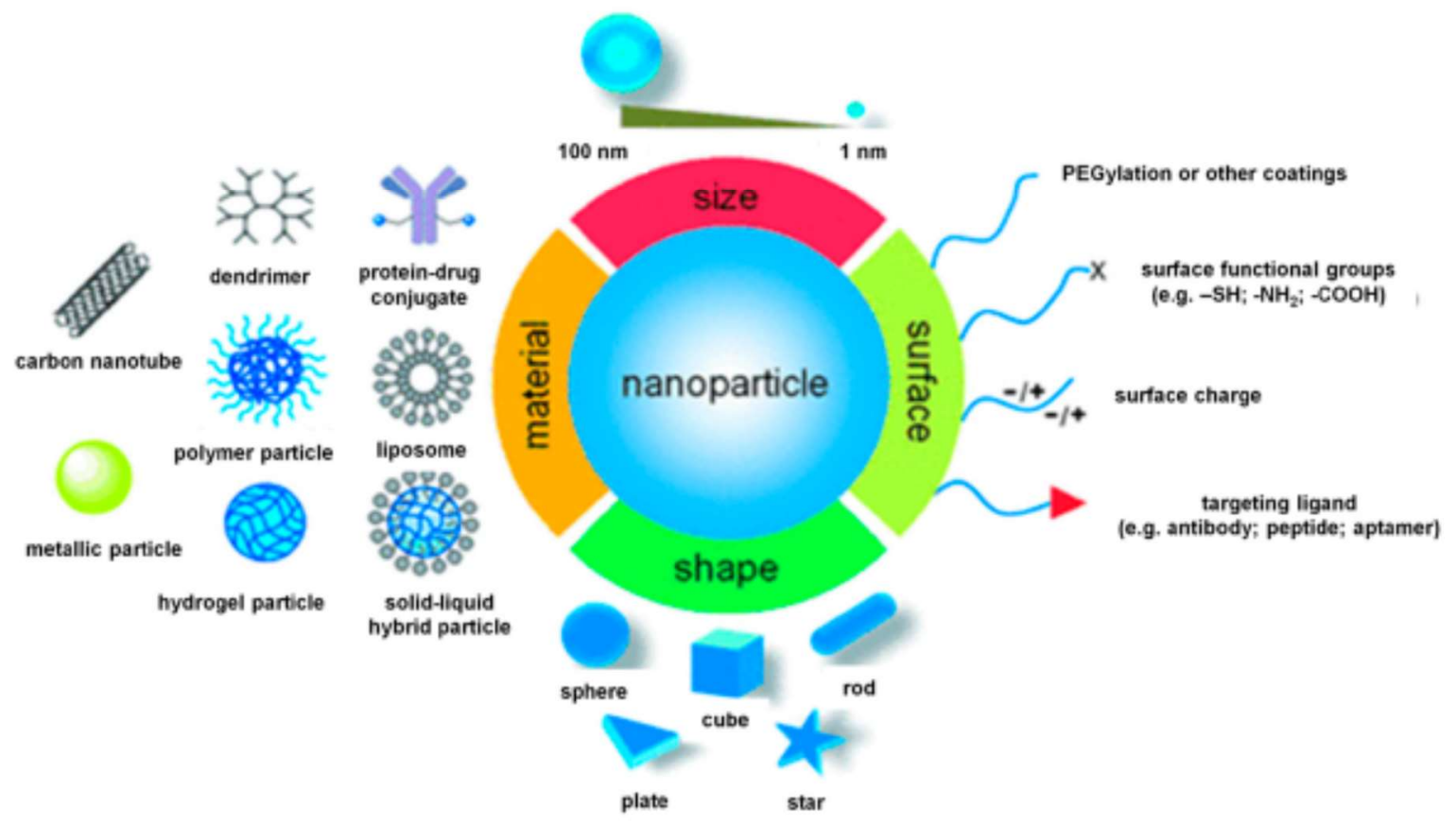

(b)

Figure 3 (a) Different kinds of MNPs functionalization (A) core/shell type MNPs; (B) MNPs functionalized with polymers; (C) MNPs enveloped in polymeric films; (D) Heterodimer MNPs; (E) MNPs functionalized with lipids mono layers and bi layers [25, 48]; (b) A summarized illustration on the kinds, functionalities and shapes for MNPs so that they are exploited as drug delivery carriers in cancerous therapeutic along with the demonstration of biophysicochemical properties. [49]. 


\section{Results and Discussion}

\subsection{Lung Cancer Detection and Monitoring}

The implication of microelectronics in sensor technology is advantageous in the development of devices that are adequately perceptive to sense biomarkers of lung cancer. In the recent years, numerous biosensors have been demonstrated for the detection of cancer-associated proteins. Simultaneously, the growing significance is towards the investigation of volatile metabolites that could be directly checked from the breath. An assessment of the state-of-the-art of volatile compound sensors and biosensors has been reviewed by R. Capuano [51]. Amongst the lung cancer markers, the worth revealing are: (1) the cytokeratin fragment 21- (CYFRA21-1) and (2) the neuron-specific enolase (NSE). These markers are well known for differentiating the two foremost kinds of lung cancer: NSCLC and SCLC [52]. The identification of these molecules can be recognized in serum containing standardized immunosorbent assays. However, such techniques involve labelling of the target molecule for their identification. The immunoselective interactions can be detected from the rapid label-free method of detection. Cheng et al. established a biosensor based on field effect transistor (FET) where the human antigen CFYFRA21-1 and neuron-specific enolase are bonded together on the sensitive surface [53]. The detection of these markers is limited to concentrations of about $1 \mathrm{ng} / \mathrm{mL}$ due to the absence of labeling method and the high device sensitivity. Apart from the brilliant consequences obtained, the biosensor technology is still not established enough for substantial use. Specifically, the transfer of the biomolecules onto a solid substrate results in the loss of its properties which is an additional limiting factor. Along with these limitations, the biosensors are have narrow lifetime and are disposable devices. However, different approach has been adopted in case of sensors based on volatile compounds. Furthermore, the description of the pathways connecting the cancerous cells and volatile compounds are relatively vague justifying the existence of various different compounds. Thus, there is non- availability of the clear indication for the particular volatile compound which is strictly linked to cancerous disease. Alternatively, volatile organic compounds are striking more attention because of its non- invasive analysis principle which has been functional at any time. In relation to biosensors, modern electronic noses have numerous benefits including its strong robustness, more stability and reversible nature. This implies that the electronic noses can be employed for several different measurements without any performance loss. Moreover, there production at large scale can also be done easily.

Combinations of biomarkers with biosensors and nanoscience have herald a new era of early diagnosis of cancer and accurate targeted drug delivery. Therefore, the significantly high importance of carcinoma theranostics has attracted the concern of various researchers to write systematic literature reviews on biomarkers [54- 58]. 
Table 2 Lung Cancer biomarkers

\begin{tabular}{|l|l|l|l|}
\hline Cancer & \multicolumn{1}{|c|}{ Markers } & Characteristics & Sample \\
\hline Lung (NSCLC) & $\begin{array}{c}\text { CEA } \\
\text { Carcinoembryonic } \\
\text { antigen }\end{array}$ & $\begin{array}{l}\text { The } \\
\text { combination of CEA } \\
\text { with tumor marker NSA } \\
\text { increases the specificity, } \\
\text { also helps in detection } \\
\text { of colon carcinoma. }\end{array}$ & Blood [59] \\
\hline Lung (SCLC) & NSE & $\begin{array}{l}\text { Improved } \\
\text { sensitivity towards } \\
\text { particular kind of lung } \\
\text { carcinoma }\end{array}$ & Blood [59] \\
\hline
\end{tabular}

The functionalization of MNPs with quantum dots provides easy detection of targeted cancerous cells with their separation and visualization [60]. The production of images with multicolor fluorescent nature and magnetic variations could be achieved by an excessive fluorescent multi-labeling in conjugation with functionalized MNPs. The immobilization of antiepithelial cell adhesion molecule (EpCAM abs) antibody results in the conjugation of targeted CTCs or circulating tumor cells [61]. However, the combination of MNPs, quantum dots and antibodies is very complicated that requires numerous steps. Consequently, a lot of research studies are carried out in order to discover simpler techniques for fabricating them.

In the report of O. Hosu et al. [25], it has been observed that the surface functionalization of MNPs provides linkage groups that permit the binding of these groups with the corresponding biomolecules. There are several bioconjugation stratagems for easy and simpler immobilization on transducers or MNPs. These strategies include physical interactions such as hydrophilichydrophobic interactions, electrostatic interaction, binding interactions while chemical interactions include, for example covalent bonding [62]. Apart from these interactions there are some particular interactions which plays significant role in the fabrication or design of biosensors. These specific interactions include interaction between protein and their detecting rudiments, like biotin-avidin linking systems and antigen-antibody binding interactions [63]. Figure 4 shows the schematic illustration of the detection of micrometastases in lung carcinoma by the combined influence of MNPs and quantum dots as given by Wang et al. [38]. The successful identification of lung carcinoma cells A549 and SPC-A-1 by quantum dots with double-labeled antibodies was noticed. As a consequence, the collection of 32 cases with NSCLC was found. There were 26 patients with enhanced CTCs and 21 patients were effectively recognized by quantum dots. Therefore, a novel technique was discovered in which CTCs was collected by MNP-pan-ck and the identification of CTCs from NSCLC patients was done simultaneously by quantum dots with double-labeled antibody. 

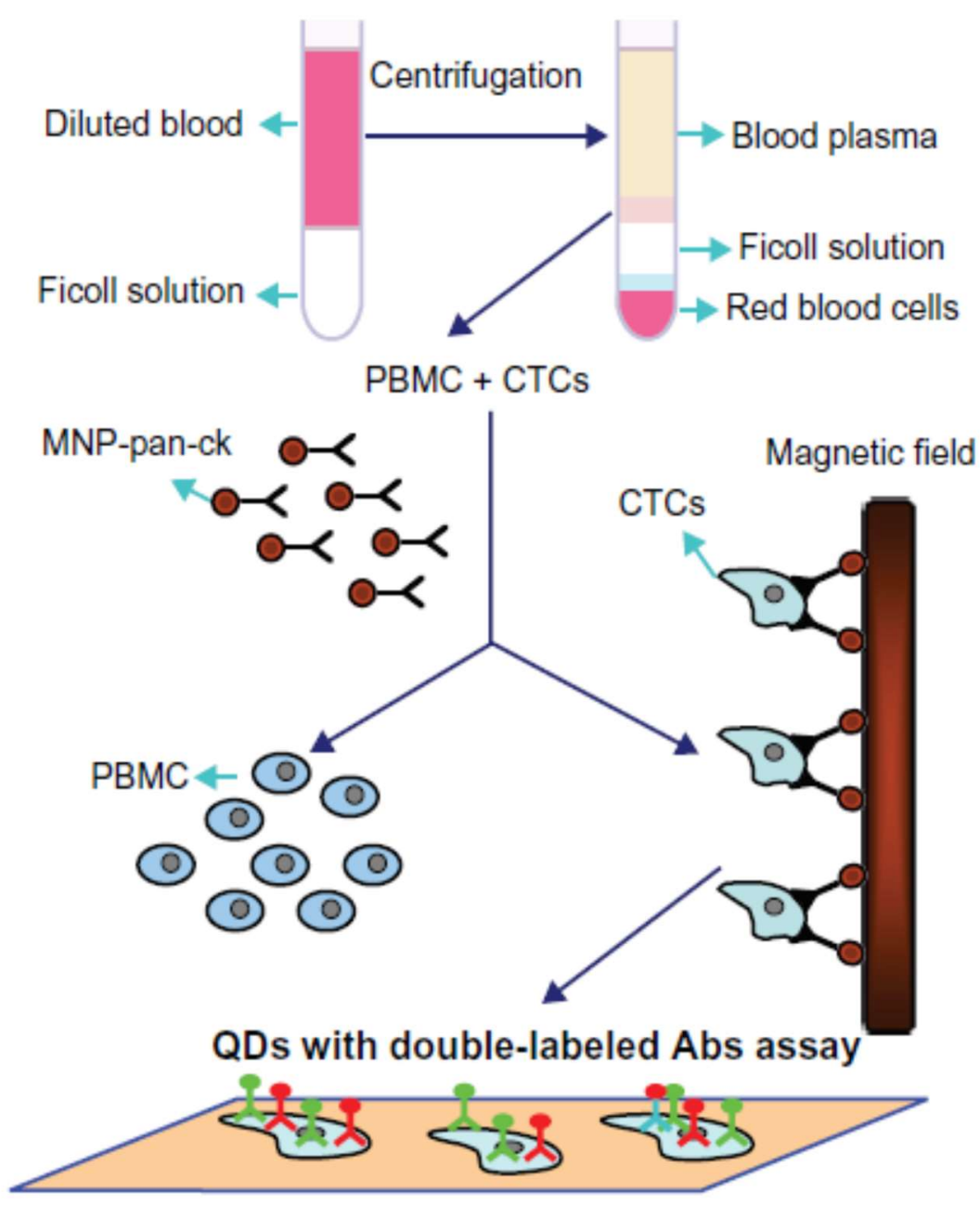

Figure 4 Diagrammatic representation of separation and recognition of circulating tumor cells with MNPs coupled pan cytokeratin antibody and quantum dots with double-labeled antibody [38]. PBMC refers to peripheral blood mononuclear cell

Table 3 The recognized inferences provided by various groups [38]

\begin{tabular}{|c|l|c|c|}
\hline Groups & MNPs enrichment (\%) & $\begin{array}{l}\text { Quantum dots recognition } \\
(\%)\end{array}$ \\
\hline NSCLC & 2 & $26(81.25 \%)$ & $21(80.77 \%)$ \\
\hline Stage I cancer & & $3(42.86 \%)$ & $2(66.67 \%)$ \\
\hline $\begin{array}{c}\text { Stage II }- \text { IV } \\
\text { Cancer }\end{array}$ & 5 & $23(92.00 \%)$ & $19(82.61 \%)$ \\
\hline $\begin{array}{c}\text { BLD } \\
\text { benign lung diseases }\end{array}$ & 6 & 0 & 0 \\
\hline Volunteers & 6 & 0 & 0 \\
\hline
\end{tabular}

Note: Cancer of stage I implies that its spread is merely in the lungs surrounded by normal tissue. Stage II, implies its spread to neighboring lymph nodes. Stage III implies that spreading of cancer to the diaphragm or chest wall near lungs; to the mediastinum or area of 
lymph nodes isolating the two lungs; or to the neck lymph nodes or lymph nodes on the other chest side. Stage IV, implies that the spreading of cancer to other body parts. The collection of stages II-IV implies metastasized cancer.

Paclitaxel (PTX) encapsulated core/shell MNPs and cold atmospheric plasma (CAP) restrain NSCLC development as illustrated by Yu et al [64]. Results established an improved drug delivery of the electrosprayed nanoparticles and homogeneous particle size distribution that had nourished release/discharge characteristics and numerous exceptional properties. An in vitro analysis revealed that PTX loaded nanoparticles together with CAP repressed the development of A549 cells more efficiently in comparison to the repression produced by individual nanoparticles.

\subsection{Role of Magnetic Nanoparticles in Lung Cancer Therapy}

Yu et al., [64] found the reduction in the drug delivery resistance might be possible by the use of CAP that could stimulate the PTX encapsulated nanoparticles in tumor cells. This increases the efficient drug medication to a point that may perhaps be conductive to abridge the drug resistance. Consequently, the incorporation of PTX encapsulated nanoparticles and CAP offers a potential means for the growth of a novel NSCLC therapeutic stratagem. The efficient and sustained drug medication of curative agents to tumor cells is permitted by PTX loaded nanoparticles. These nanoparticles were productively synthesized by a co-axial electrospraying technique. The prepared nanoparticles based drug guided materials had been evolved as an uninterrupted delivery system with an enhanced encapsulation efficacy of the antitumor agents and provide uniform size distribution, meeting the stipulations for pulmonary administration. The electrosprayed PTX encapsulated MNPs had exceptional substantial physical and chemical characteristics and displayed $\mathrm{pH}$-dependent behavior, which proves to be extremely beneficial feature in improving the development of specific drug medication at the cancerous site. The good slow discharge characteristics of PTX encapsulated electrosprayed nanoparticles had been displayed at a $\mathrm{pH}$ level of 5.5 and 7.4. At this $\mathrm{pH}$ level the MNPs persisted slow drug delivery up to $48 \mathrm{~h}$. The synergetic effect of PTX loaded MNPs and CAP displayed a remarkable enhancement in sustained PTX in vivo. The effective combined influence of PTX-loaded MNPs and CAP has proven to be a new dual carcinoma therapeutic for restraining cancerous cell growth as illustrated by the MTT assay as compared to individual treatment. CAP can also stimulate enhanced effective delivery of intracellular PTX loaded MNPs, consequently escalating the ROS production and increasing the killing effect on cancer causing cells. Therefore, the synergetic influence of targeted drug delivery and cold atmospheric plasma (CAP) may result in a standard move in cancer theranostics.

In 2013, Abdeen and Praseetha reported the generation of heat from the oscillating effect of iron oxide MNPs by the presence of magnetic field [65]. The generated heat works as the kinetic energy to considerably decrease the tumor size or cancerous tissue. Thus, this deliberately reveals that the research in the future course of time will have to focus on the MNPs pharmacokinetics in order to make certain that MNPs retain in the patient's body for longer time periods in comparison to the current period. In 2010, Fallahzadeh et al. reported that the future studies will also concentrate on the positive reaction of patient body to MNPs [66]. One of the most practiced fields of investigation involves the improvement in the carcinoma theranostics stratagems and the anticancer drugs delivery by blending functionalized superparamagnetic iron oxide nanoparticles (SPIONs) to the tumor site. The generalized killing of cancerous tissues has also been possible by SPION stimulated hyperthermia therapy. According to Anderson \& Shive 
et al., Mahmoudi et al. Soppimath et al., Inspite of their realistic biomedical applications, disruption in iron homeostasis, alteration in the genetic structures, varied cellular reactions and oxidative stress are few SPION linked toxicological features which necessarily gained consideration [67-69].

\subsection{Drug delivery}

In the past decades, SPIONs have been gaining much interest for the reason that SPIONs are usually significant in cancerous tumor imaging, therapy and drug medication [70- 72]. MNPs are found to be exceptionally appropriate for drug delivery systems. The synthesization and surface functionalization of MNPs in different sizes are carried out in order to bind various biomolecules. There are numerous problems that are required to be pondered while employing MNPs for colloidal stability, biocompatibility and carriers counting [73- 75]. The most important implication of MNPs for biomedical relevance includes targeted delivery of anticancer drugs. The drugs delivery is dependent on several essential factors related to the particle size and magnetic properties of the biocompatible nanoparticles [76, 77].

Drugs delivery systems that are based on nanoparticle may resolve the insuperable problems of curing neurological disorder [78, 79]. Schroeder et al., reported the subject of feasible immobilization with MNPs agglomerate in capillaries and the problem that still persist because of the large separation between the cancerous/pathological site and the external magnetic field [80]. Another most central issues related to chemotherapy theranostics involves the non-specific drugs that cause damage to both cancerous as well as healthy cells. In order to resolve this issue, functionalized MNPs are employed as shown in Figure 4. These MNPs delivers the anticancer agents to the main targeted regions of cancerous tissues, sustaining the nanoparticles at exactly the precise pathological sites during the release of drugs in combination with an external magnetic field. Furthermore Lima-Tenorio et al., illustrated a controlled drug delivery system on basis of MNPs that is capable of upholding the drug levels up to desired concentration range for conducting cancer treatment, this in turn curbs the over dosage and reduces the adverse effects [81].

Noteworthy progression in genetic therapy and rectification has been performed by attaching a viral vector carrying a complementary gene to the MNPs surface. This allows the modification of genetic disorders by enabling magnetic gene transfection with the complementary gene attached to MNPs surface carrying viral vector [82]. This course of action is well known as magnetofection or magnetic transfection process of genetic therapy. This therapy has meant to cure lung cancer, blood and gastrointestinal malignancies.

Current research have revealed that the growing biomedical techniques which displays immense prospective for carcinoma theranostics is the CAP with different reactive genus including reactive nitrogen, reactive oxygen and ultraviolet photons. These are the promising antipathological agents. There exists probable synergy between two outlooks. The multiple potential mechanisms of cytotoxic effect induce apoptosis/necrosis. The integration of iron oxide MNPs and CAP with their synergetic effect could indistinguisbly enhances targeted efficiency on the lung cancer theranostics [25].

SPIONs are expansively employed as a magnetic resonance imaging or MRI distinguishable agent. The utilization of SPIONs is broadly applied as a drug delivery agent or carrier in carcinoma therapeutic purpose. Iron oxide based MNPs were long exercised for different biomedical functions including drug carriers, magnetic hyperthermia, MRI imaging and cancer therapeutic agents in lung cancer [83-86]. The implication of iron oxide based MNPs has 
currently been demonstrated by Wang et al., in MRI and ultrasound ablation therapy as shown in Figure 3 [83]. The authors have demonstrated in vitro and in vivo rat xenograft model of human lung carcinoma (H460) and produced an epidermal growth factor receptor (EGFR), a protein found on the healthy and cancer cells surface namely PEGylated MNPs targeted for delivery of drugs at precise location and its imaging to lung cancer. Another study involves the magnetic hyperthermia in lung cancer as elaborated by Sadhuka et al. which showed the practice of inhalable EGFR-targeted MNPs for hyperthermia [85]. The authors displayed the targeting action of EGFR augmented the tumor retention of iron oxide based MNPs. Furthermore, it was observed that the foremost inhibition of growth in lung tumor in in vivo orthotropic lung tumor model was caused due to magnetic hyperthermia by EGFR-targeted iron oxide MNPs.

Magnetic hyperthermia involves the generation of heat by the usage of superparamagnetic materials or SPIONs through an external alternating magnetic fields (AMF). This method of removal of lung tumor is a non-invasive nature. In 2013, Sadhukha et al., exemplified that this procedure of lung cancer treatment is suitable for addressing an on demand question as it discloses the reasonably inert behavior of the magnetic materials without applying external magnetic field and their high efficiency in the presence of AMF.

In the year 2015, Obaidat et al. have noticed the magnetic hyperthermia as a local therapeutic technique for the tumor confiscation from the body. This therapy utilizes MNPs for heating the cancerous cells and initiating a non reversible impairment to the tumor sites and the surrounding tissues (Gorjikhah et al. 2016, Obaidat et al. 2015) [87, 88]. The oscillations in the MNPs are generated when MNPs approach the tumor sites and the present magnet induces vibrations into these nanoparticles. These oscillations in turn heat up the cancerous tumor to about $42^{\mathrm{c}} \mathrm{C}$ to $46^{\circ} \mathrm{C}$ slightly above the normal temperature of human body $37^{\circ} \mathrm{C}$ (Asin et al. 2012b, Obaidat et al. 2015) [87, 89]. This temperature is enough to eradicate the tumor cells and curb the after effects of the damaged cancerous cells. However, if the MNPs are unable to approach the tumor sites then the treatment may not be reasonable or effectual (Asin et al. 2012b, Cervadoro et al. 2013, Gorjikhah et al. 2016, Landi 2013, Obaidat et al. 2015) [87-91]. There are numerous metallic nanoparticles that exhibit magnetic properties however, Iron oxide MNPs are most favored choice in cancer theranostics (Rivas et al. 2012) [92]. The iron oxide MNPs are established nanoparticles with non toxicity and biocompatible nature. Such characteristics are vital as they assure patient comfortability during lung cancer treatment. Furthermore, the functioning of iron oxide MNPs is extravagant by applying the magnetic force in order to generate heat in the targeted site during carcinoma therapy (Disch et al. 2012) [93]. Before the transmission of MNPs to targeted tumor sites, these MNPs are well surface functionalized by a special coating material specifically the aminosilane (Rivas et al. 2012) [92]. This special coating is provided in such a way that it ensures the movement of targeted tumor sites by MNPs without spanning to other body parts that may results other complexities occurring during hyperthermia process. Nanotechnology is an escalating forte which is revolutionizing the carcinoma theranostics and prevention (Babu et al. 2013) [94]. The blooming synthesization and augmentation of the theranostics efficiency of various multiple anticancerous agents have been promised by the nanoscale medication systems (Babu et al. 2013, Jiang et al. 2013) [94, 95]. Also, the advanced biocompatibility of these nanoparticles ensures the nucleic acid fortification from deprivation and offers the efficient delivery of the therapeutic genes to the cancerous cells. This formulates the nanoscale particles as an ultimate system for drug carriers (Akbarzadeh et al. 2012b) [96]. Furthermore, there are still numerous issues related to different therapies based on nanoparticles and only few of them have pointed towards clinical achievement. The difficulties 
lie in the identification of ideal drug carrier systems for various newly discovered drugs with miscellaneous physiological characteristics and changeable theranostics levels (Gudoshnikov et al. 2012, Gudoshnikov et al. 2013) [97, 98].

\subsection{Direct injection of MNP into the tumor}

Recently, the MNPs delivery is established on the basis of unswerving intra-tumoral injection as reported in the year 2015, by Gobbo et al. which restricts its clinical applicability [99]. As provided in the research work of Gupta and Maier Hauff in the year 2005 and 2011, the advancement in the improvement of tumor targeting by the functionalized SPIONs provides the in vivo delivery of SPIONs to the specific tumor cells [100, 101].

\section{Conclusion}

The prevention of the hazardous effects of the lung cancer necessitates its timely prognosis and successful theranostics. The numerous challenges included in the traditional methodological way of diagnosing and treating lung cancer are overcome by the usage of nanoparticles. Nanoparticles in attendance, opens the opportunity for assuring the early prognosis and effective therapies for lung carcinoma. However, there are some noteworthy barriers involved in the usage of nanoparticles for tumor detection and treatment, progression in this area is being made, and in the upcoming world the usage of nanotechnology will prove to be the best stratagem for prognosing and curing lung cancer. Personalized cancer treatments or theranostics are significantly proof based. Herein, a prescription of an individual warrants the right and accurate treatment at the approved time so that it leads to remarkable efficiency and recovery of patient's condition and reduction in medical and treatment expenses. Nanotechnology based carrier platform provides the precise delivery of drugs and imaging agents to the targeted pathological sites of the patient body by theranostics medicines. The tremendously high benefits of nanomaterials have proven to be tools for biomedicine areas and are now employing in the clinical applications. The functionalization, surface encapsulation and supplementary formulation of nanomaterials can surmount the problems related to solubility, dispersion, stability attributed to their attractive physical properties. The delivery of appropriate medicines to the targeted regions and its prognosis has been possible by the synergetic effect of integrating drugs, ligands, imaging agents and biomolecules into formulated nanoparticles. Another characteristic feature of nanoparticles that helps in the high therapeutic dosage is the large surface area provided by nanosize particles. The reduction in the nonspecific toxicity has been possible by targeting the specific cancerous cells and providing the delivery of drugs at that specific site by nanoparticle- therapeutic payloads via passive or active agents.

Despite its numerous advantages, there are many unresolved challenges. These unsolved scale-up issues include production of economical status through funding, the drug pharmacokinetics and the specific target imaging. The clinical theranostics of lung cancer requires handling of additional issues and hurdles related to nanotoxicity and regulatory guidelines need to be followed. Though nanomedicine has reached great heights but still is not employed up to its maximal influence in lung malignancies and other hazardous diseases. With the beginning of immunohistochemistry, stronger sequencing methods and proteomic techniques, a development in the understanding of cancerous mechanisms and the recognition of novel exceptional biomarkers are on future course. The generation of new road for understanding the cancer mechanism can be made in future by opening greater funding sources for multi-center longitudinal research and the beginning of novel genomic strategies and programmes. The 
advancement in the theranostics stratagems based on radiomics will widen using insightful learning from the present efforts to the predictable future. Progression of therapeutic and diagnostic machineries for vulnerable and perceptible analysis of prognostic biomarkers, diagnostic capability, high resolution quantification of tumor at earliest stage and early respond to cancerous treatment will be reliable on the operation and management of new supporting machineries (for example: artificial intelligence). The incorporation of all the advancement made in these associated domains jointly as carcinoma prognostics is primed to modernize the prospects of theranostics for the eventual obliteration of lung cancer.

\section{CREdiT Authorship Contribution Statement}

Ayushi Rastogi: Resources, Writing - original draft, Writing - review \& editing. Pankaj Tripathi: Conceptualization, Resources, Writing - original draft, Writing - review \& editing. Fanindra Pandey: Writing - original draft, review \& editing. Rajiv Manohar: Conceptualization, Validation, Methodology, Resources, Writing - review \& editing. Shri Singh: Conceptualization, Validation, Methodology, Resources, Writing - review \& editing.

\section{Disclosure Statement}

All authors report no conflict of interest.

\section{Acknowledgement}

A.R is thankful to UGC (F-25-1/2014-15(BSR)/7-177/2007/BSR) New Delhi for providing financial assistance in the form of UGC-BSR Fellowship. R.M is thankful to UGC for UGC MID career award and Centre of Excellence at APJ Abdul Kalam Centre, University of Lucknow.

\section{References}

1. Edwards, B. K; Noone, A. M; Mariotto, A. B; Simard, E. P; Boscoe, F. P; Henley, S. J; Jemal, A.; Cho, $\underline{H}$.; Anderson, R.N.; Kohler, B. A.; Eheman, C. R.; Ward, E. M. Annual Report to the Nation on the status of cancer, 1975 to 2010, featuring prevalence of comorbidity and impact on survival among persons with lung, colorectal, breast, or prostate cancer. Cancer. 2014, 120 (9), 1290-314.

2. Sivarajakumar, R; Mallukaraj, D; Kadavakollu, M; Neelakandan, N; Chandran, S; Bhojaraj, S; Karri, V. V. S. R. Nanoparticles for the Treatment of Lung Cancers. $J$ Young Pharm, 2018, 10(3), 276-281.

3. Armitage, P.; Doll, R. The age distribution of cancer and a multi-stage theory of carcinogenesis. Br. J. Cancer. 1954, 8(1), 1.

4. Collins, L. G.; Haines, C.; Perkel, R.; Enck, R. E. Lung cancer: diagnosis and management. Am. Fam. Physician. 2007, 75, 56-63.

5. Howlader, N.; Noone, A. M.; Krapcho, M.; Garshell, J.; Neyman, N.; Altekruse, S. F.; Kosary, C. L.; Yu, M.; Ruhl, J.; Tatalovich Z.; Cho, H.; Mariotto, A.; Lewis, D. R.; Chen, H. S.; Feuer, E. J.; Cronin, K. A. (eds) SEER Cancer Statistics Review, 1975 - 2010. [Based on the November 2012 SEER data submission, posted to the SEER web site, April 2013.]. Bethesda, MD: National Cancer Institute.

6. Bjerager, M.; Palshof, T.; Dahl, R.; Vedsted, P.; Olesen, F. Delay in diagnosis of lung cancer in general practice. Br. J. Gen. Pract. 2006, 56, 863-868.

7. Mackillop, W. J. Killing time: the consequences of delays in radiotherapy. Radiother. Oncol. 2007, 84, 1-4. doi: 10.1016/j.radonc.2007.05.006 
8. Aberle, D. R.; Adams, A. M.; Berg, C. D.; Black, W. C.; Clapp, J. D.; Fagerstrom, R. M.; Gareen, I. F.; Gatsonis, C.; Marcus, P. M.; Sicks, J. D. Reduced lung-cancer mortality with low-dose computed tomographic screening. N. Engl. J. Med. 2011, 365, 395-409. doi: 10.1056/NEJMoa1102873

9. Wildstein, K. A.; Faustini, Y.; Yip, R.; Henschke, C. I.; Ostroff, J. S. Longitudinal predictors of adherence to annual follow-up in a lung cancer screening programme. $J$. Med. Screen. 2011, 18, 154-159. doi: 10.1258/jms.2011.010127

10. Goulart, B. H.; Bensink, M. E.; Mummy, D. G.; Ramsey, S. D. Lung cancer screening with low-dose computed tomography: costs, national expenditures, and costeffectiveness. J. Natl. Compr. Canc. Netw. 2012, 10, 267-275.

11. Brenner, D. J. Radiation and chest CT scans: are there problems? What should we do? Chest. 2012, 142, 549-550. doi: 10.1378/chest.12-0490

12. McCunney, R. J., and Li, J. Radiation risks in lung cancer screening programs. Chest. 2014, 145, 618-624. doi: 10.1378/chest.13-1420

13. Anari, E.; Akbarzadeh, A.; Zarghami, N. Chrysin-loaded PLGA-PEG nanoparticles designed for enhanced effect on the breast cancer cell line. Artif. Cells Nanomed. Biotechnol. [Epub ahead of print]. doi: 10.3109/21691401.2015.1029633.

14. Bray, F.; Ferlay, J.; Soerjomataram, I.; Siegel, R.L.; Torre, L.A.; Jemal, A. Global cancer statistics 2018: GLOBOCAN estimates of incidence and mortality worldwide for 36 cancers in 185 countries. CA Cancer J. Clin. 2018, 68, 394-424.

15. Ferlay, J.; Soerjomataram, I.; Dikshit, R.; Eser, S.; Mathers, C.; Rebelo, M.; Parkin, D.M.; Forman, D.; Bray, F. Cancer incidence and mortality worldwide: Sources, methods and major patterns in GLOBOCAN 2012. Int. J. Cancer 2015, 136, E359-E386.

16. Rudokas, M.; Najlah, M.; Alhnan, M. A.; Elhissi, A. Liposome Delivery Systems for Inhalation: A Critical Review Highlighting Formulation Issues and Anticancer Applications. Med. Princ. Pract. 2016, 25, 60-72.

17. Miller, K. D.; Siegel, R. L.; Lin, C. C.; Mariotto, A. B.; Kramer, J. L.; Rowland, J.H.; Stein, K. D.; Alteri, R.; Jemal, A. Cancer treatment and survivorship statistics, 2016. CA Cancer J. Clin. 2016, 66, 271-289.

18. Munaweera, I.; Shi, Y.; Koneru, B.; Saez, R.; Aliev, A.; Di Pasqua A. J.; Balkus, K. J. Jr. Chemoradiotherapeutic magnetic nanoparticles for targeted treatment of nonsmall cell lung cancer. Mol. Pharm. 2015, 12, 3588-3596.

19. Tabatabaei Mirakabad, F. S.; Akbarzadeh, A.; Milan, M.; Zarghami, N.; TaheriAnganeh, M.; Zeighamian, V.; Badrzadeh, F.; Rahmati-Yamchi, M. A Comparison between the cytotoxic effects of pure curcumin and curcumin- loaded PLGA-PEG nanoparticles on the MCF-7 human breast cancer cell line. Artif. Cells Nanomed. Biotechnol. 2016, 44, 423-430.

20. Wong, M. C. S.; Lao, X. Q.; Ho, K. F.; Goggins, W. B.; Tse, S. L. A. Incidence and mortality of lung cancer: global trends and association with socioeconomic status. Sci. Rep. 2017, 7, 14300. doi: 10.1038/s41598-017-14513-7

21. Van der Aalst, C. M.; de Koning, H. J. Biochemical verification of the self-reported smoking status of screened male smokers of the Dutch-Belgian randomized controlled lung cancer screening trial. Lung Cancer. 2016, 94, 96-101.

22. WHO. Cancer; WHO: Geneva, Switzerland, 2019. 
23. Cohen, Z. R.; Ramishetti, S.; Peshes-Yaloz, N.; Goldsmith, M.; Wohl, A.; Zibly, Z.; Peer, D. Localized RNAi therapeutics of chemoresistant grade IV glioma using hyaluronangrafted lipid-based nanoparticles. ACS Nano. 2015, 9, 1581-1591.

24. Mukherjee, A.; Paul, M.; Sudip Mukherjee, Recent Progress in the Theranostics Application of Nanomedicine in Lung Cancer. Cancers. 2019, 11, 597; doi:10.3390/cancers 11050597

25. Hosu, O.; Tertis, M.; Cristea, C. Implication of Magnetic Nanoparticles in Cancer Detection, Screening and Treatment. Magnetochemistry. 2019, 5, 55; doi:10.3390/magnetochemistry5040055

26. Tapeinos, C. Magnetic nanoparticles and their Bioapplications. In Smart Nanoparticles for Biomedicine. Elsevier: Amsterdam, the Netherlands. 2018, 131-142, ISBN 9780128141564.

27. Xianyu, Y.; Wang, Q.; Chen, Y. Magnetic particles-enabled biosensors for point-of-care testing. TrAC Trends in Anal. Chem. 2018, 106, 213-224.

28. Kralj, S.; Rojnik, M.; Kos, J.; Makovec, D. Targeting EGFR-overexpressed A431 cells with EGF-labeled silica-coated magnetic nanoparticles. J. Nanoparticle Res. 2013, 15, 1666.

29. Shanta Singh, N.; Kulkarni, H.; Pradhan, L.; Bahadur, D. A multifunctional biphasic suspension of mesoporous silica encapsulated with $\mathrm{YVO}(4): \operatorname{Eu}(3+)$ and $\operatorname{Fe}(3) \mathrm{O}(4)$ nanoparticles: synergistic effect towards cancer therapy and imaging. Nanotechnology. 2013, 24, 065101.

30. Fang, C.; Kievit, F. M.; Veiseh, O.; Stephen, Z. R.; Wang, T.; Lee, D.; Ellenbogen, R. G.; Zhang, M. Fabrication of magnetic nanoparticles with controllable drug loading and release through a simple assembly approach. J. Control. Release. 2012, 162, 233 - 241.

31. Yallapu, M. M.; Othman, S. F.; Curtis, E. T.; Curtis, E. T.; Gupta, B. K.; Jaggi, M.; Chauhan, S. C. Multi-functional magnetic nanoparticles for magnetic resonance imaging and cancer therapy. Biomaterials. 2011, 32, $1890-1905$.

32. Guo, D.; Wu C, Hu H, Wang X, Li X, Chen B. Study on the enhanced cellular uptake effect of daunorubicin on leukemia cells mediated via functionalized nickel nanoparticles. Biomed. Mater. 2009, 4, 025013.

33. Alexiou, C.; Tietze, R.; Schreiber, E.; Lyer, S. Nanomedicine: magnetic nanoparticles for drug delivery and hyperthermia - new chances for cancer therapy. Bundesgesundheitsblatt Gesundheitsforschung Gesundheitsschutz. 2010, 53, 839 - 845.

34. Durr, S.; Lyer, S.; Mann.; Janko, C.; Tietze, R. Schreiber E, Herrmann M, Alexiou C. Real-time cell analysis of human cancer cell lines after chemotherapy with functionalized magnetic nanoparticles. Anticancer Res. 2012, 32, 1983 - 1989.

35. Alexiou, C.; Diehl, D.; Henninger, P.; Iro, H.; Rockelein, R.; Schmidt, W.; Weber, H. A high field gradient magnet for magnetic drug targeting. IEEE T. Appl. Supercon. 2006, $16,1527-1530$.

36. Tietze, R.; Rahn, H.; Lyer, S.; Schreiber, E.; Mann, J.; Odenbach, S.; Alexiou, C. Visualization of superparamagnetic nanoparticles in vascular tissue using XmuCT and histology. Histochem. Cell Biol. 2011, 135, 153 - 158.

37. Seliger, C.; Jurgons, R.; Wiekhorst, F.; Eberbeck, D.; Trahms, L.; Iro, H.; Alexiou, C. In vitro investigation of the behaviour of magnetic particles by a circulating artery model. $J$. Magn. Magn. Mater. 2007, 311, 358 - 362. 
38. Wang, Y.; Zhang, Y.; Du, Z.; Wu, M.; Zhang, G. Detection of micrometastases in lung cancer with magnetic nanoparticles and quantum dots. Intl. J. Nanomedicine. 2012,7, 2315-2324.

39. Chen, Z.; Wu, C.; Zhang, Z.; Wu, W.; Wang, X.; Yu, Z. Synthesis, functionalization, and nanomedical applications of functional magnetic nanoparticles. Chin. Chem. Lett. 2018, 29, 1601-1608.

40. Cristea, C.; Tertis, M.; Galatus, R. Magnetic nanoparticles for antibiotics detection. Nanomaterials. 2017, 7, 119.

41. Rocha-Santos, T.A.P. Sensors and biosensors based on magnetic nanoparticles. $\operatorname{Tr} A C$ Trends Anal. Chem. 2014, 62, 28-36.

42. Xu, J. K.; Zhang, F. F.; Sun, J. J.; Sheng, J.; Wang, F.; Sun, M. Bio and nanomaterials based on $\mathrm{Fe}_{3} \mathrm{O}_{4}$. Molecules. 2014, 19, 21506-21528.

43. Liu, C.; Zou, B.; Rondinone, A. J.; Zhang, Z. J. Reverse micelle synthesis and characterization of superparamagnetic $\mathrm{MnFe}_{2} \mathrm{O}_{4}$ spinel ferrite nanocrystallites. J. Phys. Chem. B. 2000, 104, 1143-1145.

44. Karade, V. C.; Dongale, T. D.; Sahoo, S. C.; Kollu, P.; Chougale, A. D.; Patil, P. S.; Patil, P. B. Effect of reaction time on structural and magnetic properties of greensynthesized magnetic nanoparticles. J. Phys. Chem. Solids, 2018, 120, 161-166.

45. Magdziarz, A.; Colmenares, J.C. In situ coupling of ultrasound to electro-and photodeposition methods for materials synthesis. Molecules. 2017, 22, 216.

46. Abd Elrahman, A. A.; Mansour, F. R. Targeted magnetic iron oxide nanoparticles: Preparation, functionalization and biomedical application. J. Drug Deliv. Sci. Technol. 2019, 52, 702-712.

47. Liu, G.; Li, R.-W.; Chen, Y. Magnetic Nanoparticle for Biomedicine Applications. Nanotechnol. Nanomed. Nanobiotechnol. 2015, 2, 1-7.

48. Kudr, J.; Haddad, Y.; Richtera, L.; Heger, Z.; Cernak, M.; Adam, V.; Zitka, O. Magnetic nanoparticles: From design and synthesis to real world applications. Nanomaterials. 2017, 7, 243.

49. Sun, T.; Zhang, Y. S.; Pang, B.; Hyun, D. C.; Yang, M.; Xia, Y. Engineered nanoparticles for drug delivery in cancer therapy. Angew. Chem. Int. Ed. 2014, 53, $12320-12364$.

50. Gangwar, A.; Varghese, S. S.; Meena, S. S.; Prajapat, C. L.; Gupta, N.; Prasad, N. K. Fe $3 \mathrm{C}$ nanoparticles for magnetic hyperthermia application. J. Magn. Magn. Mater. 2019, 481, 251-256.

51. Capuano, R.; Catini, A.; Paolesse, R.; Natale, C. D. Sensors for Lung Cancer Diagnosis, J. Clin. Med. 2019, 8, 235. doi:10.3390/jcm8020235

52. Hatzakisa, K. D.; Froudarakisa, M. E.; Bourosa, D.; Tzanakisa, N.; Karkavitsasb, N.; Siafakasa, N. M. Prognostic value of serum tumor markers in patients with lung cancer. Respiration. 2002, 69, 25-29.

53. Cheng, S.; Hideshim, S.; Kuroiwa, S.; Nakamishi, T.; Osaka, T. Label-free detection of tumor markers using FET based biosensores for lung cancer diagnosis. Sensors and Actuaor, B: Chem. 2015, 212, 329-334.

54. Choi, Y. E.; Kwak, J. W.; Park, J. W. Nanotechnology for Early Cancer Detection, Sensors. 2010, 10, 428-455. doi:10.3390/s100100428

55. Kumar, S.; Mohan, A.; Guleria, R. Biomarkers in cancer screening, research and detection: present and future: a review. Biomarkers. 2006, 11, 385-405. 
56. Hanash, S.; Pitteri, S.; Faca, V. Mining the plasma proteome for cancer biomarkers Nature. 2008, 452, 571-579.

57. Saerens, D.; Huang, L.; Bonroy, K.; Muyldermans, S. Antibody fragments as probe in biosensor development. Sensors. 2008, 8, 4669-4686.

58. D'haeseleer, P. How does DNA sequence motif discovery work? Nat. Biotechnol. 2006, 24, 959-961.

59. Molina, R.; Auge, J.; Escudero, J.; Marrades, R.; Vinolas, N.; Carcereny, E.; Ramirez, J.; Filella, X. Mucins CA 125, CA 19.9, CA 15.3 and TAG-72.3 as tumor markers in patients with lung cancer: comparison with CYFRA 21-1, CEA, SCC and NSE. Tumor Biol. 2008, 29, 371-380.

60. Wang, D.; He, J.; Rosenzweig, N.; Rosenzweig, Z. Superparamagnetic Fe2O3 beads $-\mathrm{CdSe} / \mathrm{ZnS}$ quantum dots core-shell nanocomposite particles for cell separation. Nano Lett. 2004, 4, 409-413.

61. Maeda, Y.; Yoshino, T.; Matsunaga, T. Novel nanocomposites consisting of in vivobiotinylated bacterial magnetic particles and quantum dots for magnetic separation and fluorescent labeling of cancer cells. J. Mater. Chem. 2009, 19, 6361-6366.

62. Kouassi, G. K.; Irudayaraj, J. Magnetic and gold-coated magnetic nanoparticles as a DNA sensor. Anal. Chem. 2006, 78, 3234-3241.

63. Chen, Y. T.; Kolhatkar, A. G.; Zenasni, O.; Xu, S.; Lee, T. R. Biosensing using magnetic particle detection techniques. Sensors. 2017, 17, 2300.

64. Yu, H.; Wang, Y. H.; Wang, S.; Li, X.; Li, W.; Ding, D.; Gong, X.; Keidar, M.; Zhang, W. Paclitaxel- Loaded Core-shell Magnetic Nanoparticles and Cold Atmospheric Plasma Inhibit Non-small Cell Lung Cancer Growth. ACS Appl. Mater. Interfaces. DOI: 10.1021/acsami.8b16487

65. Abdeen, S.; Praseetha, P. K. Diagnostics and treatment of metastatic cancers with magnetic nanoparticles. J. Nanomed. Biother. Discov. 2013, 3,115.

66. Fallahzadeh, S.; Bahrami, H.; Akbarzadeh, A.; Tayarani, M. High-isolation dualfrequency operation patch antenna using spiral defected microstrip structure. IEEE Antenn. Wirel. Propagat. Lett. 2010, 9, 122-124.

67. Anderson, J. M.; Shive, M. S. Biodegradation and biocompatibility of PLA and PLGA microspheres. Adv. Drug Deliv. Rev. 2012, 64, 72-82.

68. Mahmoudi, M.; Laurent, S.; Shokrgozar, M. A.; Hosseinkhani, M. Toxicity evaluations of superparamagnetic iron oxide nanoparticles: cell "vision" versus physicochemical properties of nanoparticles. ACS Nano. 2011, 5, 7263-7276.

69. Soppimath, K. S.; Aminabhavi, T. M.; Kulkarni, A. R.; Rudzinski, W. E. Biodegradable polymeric nanoparticles as drug delivery devices. J. Control Release. 2001, 70, 1-20.

70. McCarthy, J. R.; Weissleder, R. Multifunctional magnetic nanoparticles for targeted imaging and therapy. Adv. Drug Deliv. Rev. 2008, 60, 1241-1251.

71. Petters, C.; Irrsack, E.; Koch, M.; Dringen, R. Uptake and metabolism of iron oxide nanoparticles in brain cells. Neurochem. Res. 2014, 39, 1648-1660.

72. Salloum, M.; Ma, R.; Zhu, L. Enhancement in treatment planning for magnetic nanoparticle hyperthermia: optimization of the heat absorption pattern. Int. J. Hyperthermia. 2009, 25:309-321.

73. Faraji, M.; Yamini, Y.; Rezaee, M. Magnetic nanoparticles: synthesis, stabilization, functionalization, characterization, and applications. J. Iranian Chem. Soc. 2010, 7, 1-37. 
74. Furlani, E. P. Magnetic biotransport: analysis and applications. Mater. 2010, 3, 24122446.

75. Tartaj, P.; Morales, M. D. D.; Veintemillas- Verdaguer, S.; Gonzalez - Carreno, T.; Serna, C. J. The preparation of magnetic nanoparticles for applications in biomedicine. $J$. Phys. D Appl. Phys. 2003, 36, R182-R197.

76. Akbarzadeh, A.; Samiei, M.; Davaran, S. Magnetic nanoparticles: preparation, physical properties, and applications in biomedicine. Nanoscale Res. Lett. 2012a, 7, 1-13.

77. Sun, C.; Lee, J. S. H.; Zhang, M. Magnetic nanoparticles in MR imaging and drug delivery. Adv. Drug Deliv. Rev. 2008, 60, 1252-1265.

78. Nasrabadi, H. T.; Abbasi, E.; Davaran, S.; Kouhi, M.; Akbarzadeh, A. Bimetallic nanoparticles: Preparation, properties, and biomedical applications. Artif. Cells Nanomed. Biotechnol. 2016, 44, 376-380.

79. Kreuter, J. Nanoparticulate systems for brain delivery of drugs. Adv. Drug Deliv. Rev. 2001, 47, 65-81.

80. Schroeder, U.; Sommerfeld, P.; Ulrich, S.; Sabel, B. A. Nanoparticle technology for delivery of drugs across the blood-brain barrier. J. Pharm. Sci. 1998, 87, 1305-1307.

81. Lima-Tenorio, M. K.; Gomez Pineda, E. A.; Ahmad, N. M.; Fessi, H.; Elaissari, A.; Magnetic nanoparticles: in vivo cancer diagnosis and therapy. Int. J. Pharm. 2015, 493, $313-327$.

82. Issa, B.; Obaidat, I. M.; Albiss, B. A.; Haik, Y. Magnetic nanoparticles: Surface effects and properties related to biomedicine applications. Int. J. Mol. Sci. 2013, 14, 2126621305.

83. Wu, C. Y.; Chen, Y. C. Riboflavin immobilized Fe3O4 magnetic nanoparticles carried with n-butylidenephthalide as targeting-based anticancer agents. Artif. Cells Nanomed. Biotechnol. 2019, 47, 210-220.

84. Wang, F.; Chen, C.; Chen, Y.; Wang, P.; Chen, C.; Geng, D.; Li, L.; Song, T. Magnetically targeted photothemal cancer therapy in vivo with bacterial magnetic nanoparticles. Colloids Surfaces B Biointerfaces. 2018, 172, 308-314.

85. Bekaro glu, M. G.; Alemdar, A.; I, sçi, S. Comparison of ionic polymers in the targeted drug delivery applications as the coating materials on the $\mathrm{Fe}_{3} \mathrm{O}_{4}$ nanoparticles. Mater. Sci. Eng. C. 2019, 103, 109838.

86. Panda, J.; Satapathy, B. S.; Majumder, S.; Sarkar, R.; Mukherjee, B.; Tudu, B. Engineered polymeric iron oxide nanoparticles as potential drug carrier for targeted delivery of docetaxel to breast cancer cells. J. Magn. Magn. Mater. 2019, 485, 165-173.

87. Obaidat, I. M.; Issa, B.; Haik, Y. Magnetic properties of magnetic nanoparticles for efficient hyperthermia. Nanomaterials. 2015, 5, 63-89.

88. Gorjikhah, F.; Davaran, S.; Salehi, R.; Bakhtiari, M.; Hasanzadeh, A.; Panahi, Y.; Emamverdy, M.; Akbarzadeh, A. Improving "lab-on-a-chip" techniques using biomedical nanotechnology: a review. Artif. Cells Nanomed. Biotechnol. 2016. doi: 10.3109/21691401.2015.1129619.

89. Asin, L.; Ibarra, M. R.; Tres, A.; Goya, G. F. Controlled cell death by magnetic hyperthermia: effects of exposure time, field amplitude, and nanoparticle concentration. Pharm. Res. 2012b, 29, 1319-1327.

90. Cervadoro, A.; Giverso, C.; Pande, R.; Sarangi, S.; Preziosi, L.; Wosik, J.; Brazdeikis, A.; Decuzzi, P. Design maps for the hyperthermic treatment of tumors with superparamagnetic nanoparticles. 2013, PLoS One. 8:e57332. 
91. Landi, G. T. Simple models for the heating curve in magnetic hyperthermia experiments. J. Magn. Magn. Mater. 2013, 326, 14-21.

92. Rivas, J.; Banobre-Lopez, M.; Pineiro-Redondo, Y.; Rivas, B.; Lopez-Quintela, M. A.; Magnetic nanoparticles for application in cancer therapy. J. Magn. Magn. Mater. 2012, 324, 3499-3502.

93. Disch, S.; Wetterskog, E.; Hermann, R. P.; Wiedenmann, A.; Vainio, U.; SalazarAlvarez, G.; Bergstr€om, L.; Br€uckel, T. Quantitative spatial magnetization distribution in iron oxide nanocubes and nanospheres by polarized small-angle neutron scattering. New J. Phys. 2012, 14, 013025.

94. Babu, A.; Templeton, A. K.; Munshi, A.; Ramesh, R. Nanoparticle-based drug delivery for therapy of lung cancer: progress and challenges. J. Nanomater. 2013, 863951.

95. Jiang, L.; Li, X.; Liu, L.; Zhang, Q. Thiolated chitosan-modified PLA-PCLTPGS nanoparticles for oral chemotherapy of lung cancer. Nanoscale Res. Lett. 2013, 8, 1-11.

96. Akbarzadeh. A.; Samiei, M.; Joo, S. W.; Anzaby, M.; Hanifehpour, Y.; Nasrabadi, H. T.; Davaran, S. Synthesis, characterization and in vitro studies of doxorubicin-loaded magnetic nanoparticles grafted to smart copolymers on A549 lung cancer cell line. $J$. Nanobiotechnol. 2012b, 10, 46-58.

97. Gudoshnikov, S. A.; Liubimov, B. Y; Usov, N. A. Hysteresis losses in a dense superparamagnetic nanoparticle assembly. AIP Adv. 2012, 2, 012143.

98. Gudoshnikov S. A, Liubimov, B. Y, Sitnov, Y. S..; Skomarovsky, V. S.; Usov N. A. AC magnetic technique to measure specific absorption rate of magnetic nanoparticles. $J$. Superconduct. Novel Magn. 2013, 26, 857-860.

99. Gobbo, O. L.; Sjaastad, K.; Radomski, M. W.; Volkov, Y.; Prina-Mello, A. Magnetic nanoparticles in cancer theranostics. Theranostics. 2015, 5, 1249-1263.

100. Gupta, A. K.; Gupta, M. Synthesis and surface engineering of iron oxide nanoparticles for biomedical applications. Biomaterials. 2005, 26, 3995-4021.

101. Maier-Hauff, K.; Ulrich, F.; Nestler, D.; Niehoff, H.; Wust, P.; Thiesen, B.; Orawa, H.; Budach, V.; Jordan, A. Efficacy and safety of intratumoral thermotherapy using magnetic iron-oxide nanoparticles combined with external beam radiotherapy on patients with recurrent glioblastoma multiforme. J. Neurooncol. 2011, 103, 317-324. 